\section{NEUTROPHIL ACTIVATION AND THE ACUTE PHASE RESPONSE IN INVERMECTIN TREATED ONCHOCERCIASIS}

NJOO F.L.*, HACK C.E.**, OOSTING J.***, LUYENDIJK L.*, STILMA J.S.****, KIJLSTRA A. *, ******

KEYWORDS : onchocerciasis. invermectin. adverse reactions, elastase. lactoferrin. C-reactive protein. acute phase response. IL-6. IL-8. sera.

nchocerciasis, caused by the parasite Onchocerca volvulus is one of the most important causes of blindness worldwide. Ivermectin is a safe and effective drug for onchocerciasis treatment, however, it may induce adverse reactions. The mechanisms underlying the side effects are not known. In the present study the involvement of neutrophilic granulocytes in the development of these reactions and the acute phase response were investigated.

156 onchocerciasis patients and 13 skin snip negative endemic controls were given a single dose of ivermectin (150 $\mu \mathrm{g} / \mathrm{kg}$ bodyweight). Prior to treatment all subjects received a parasitological and clinical examination. Blood samples were collected before and 1 and 2 days after treatment. During 3 days after treatment, side effects were scored using Greene's classification system. Based on this system the patients were divided in 4 groups : without reactions, with mild, moderate and severe reactions. Elastase and lactoferrin, two neutrophil products which are released upon neutrophil activation, were measured using radioimmunoassays. IL-8, a cytokine that is known to activate neutrophils, was determined in plasma with an ELISA. C-reactive protein levels in serum were measured using an immunodiffusion assay (LCPartigen ${ }^{\circledR}$ Behring, Marburg, Germany). Interleukin-6, an important trigger to hepatocytes for the generation of acute phase proteins, was quantitated in plasma by ELISA.

After treatment, a rise of elastase levels was observed, which correlated with the severity of side effects $(p<0.02$, multivariance analysis). Also, a significant correlation between the percentage of patients with elevated elastase levels and the grade of side effects was found ( $p<0.0001$, $X^{2}$ trend test). Contrary to elastase, lactoferrin levels did not change significantly after ivermectin treatment. In none of the patients tested were elevated IL-8 levels found.

C-reactive protein levels increased in all patient groups after ivermectin treatment, but not in the control group. Again, the $\mathrm{C}$-reactive protein rise was associated with the degree of side effects ( $p<0.0001$, Kruskal-Wallis test). In $45 \%$ of the patients with raised CRP, IL- 6 levels were also elevated. Also, the percentage of patients with increased IL-6 levels correlated with the grade of adverse reactions $(p<0.0001$, $X^{2}$ trend test).

In conclusion : adverse reactions occurring after ivermectin treatment in onchocerciasis patients are related with neutrophil activation, C-reactive protein production and IL-6

* The Netherlands Ophtalmic Research Institute, Amsterdam.

** Central Laboratory of the Netherlands Red Cross Blood Transfusion Service and the Laboratory for Experimental and Clinical Immunology, University of Ammsterdam.

*** Dept. of Clinical Epidemiology and Biostatistics, University of Amsterdam

${ }^{* * * *}$ F.C. Donders Institute, Utrecht

******* Dept of Ophtalmology, The Netherlands. release. The triggers of these events however, remain to be elucidated.

\section{ACKNOWLEDGEMENTS}

his study was supported by a grant of Dr. F.P. Fischer Stichting in Utrecht.

\section{T CELL RECOGNITION AND ACTIVATION IN FILARIASIS}

YAZDANBAKHSH M.*, SARTONO E.*, ${ }^{* *}$, KRUIZE Y.C.M., KURNIAWAN A.**, SELKIRK M.E.***, MAIZELS R.M.***

KEYWORDS : filariasis. T cells. CD27. IgG4. IgE.

he association of heterogeneous immune responses with a spectrum of clinical manifestations in lymphatic filariasis has been confirmed in an area endemic for Brugian filariasis in Sumatra, Indonesia. A number of new findings have emerged due to the large number of individuals participating in the study. Microfilaremics all show profound T cell hyporesponsiveness as shown in earlier studies (King \& Nutman, 1991) but both endemic normals and elephantiasis patients could be divided into two groups based on high or low $\mathrm{T}$ cell reactivity to parasite antigens (Yazdanbakhsh et al., 1993). Measurement of all anti-filarial IgG isotypes in every individual (Kurniawan A. et al., 1993) has allowed us to assess correlations in $\mathrm{T}$ cell reactivity with antibody responses. T cell reactivity correlated negatively with anti-filarial IgG4 antibodies irrespective of clinical status. As IgG4 seems to indicate the presence of active infection, the data shows that $\mathrm{T}$ cell responses are primarily controlled by parasite load. The fact that not all elephantiasis patients show immune hyper-responsiveness to filarial antigens casts doubt on the role $\mathrm{T}$ cell hyper-reactivity might play in lymphatic pathogenesis.

In order to identify $\mathrm{T}$ cell subsets involved in lymphatic filarial infection, several approaches have been taken; cytokine release profiles and $\mathrm{T}$ cell activation markers have been studied in all clinical categories. Lymphatic filarial infection leads to an expansion of TH2 cells as determined by elevated IL4 release in both elephantiasis patients and microfilaremics versus endemic normals and non-endemic controls (Mahanty et al.,1992). Both sCD25 and sCD27 are T cell activation markers that are up-regulated upon $\mathrm{T}$ cell stimulation via CD3/TCR and subsequently releases into body fluids (Hintzen et al., 1991). Serum levels of these molecules can shed light on the in vivo activation of $\mathrm{T}$ cell subsets as they seem to be modulated differentially on distinct $\mathrm{T}$ cells. In lymphatic filariasis, both elephantiasis and microfilaremics have elevated levels of sCD27 when compared to endemic normals (Figure 1). The sCD25 levels, however, are similar in all clinical groups. This indicates that there is a filarial driven activation of a distinct $\mathrm{T}$ cell subset and this seems indeed to be reflected in the presence of elevated levels of $\mathrm{CD} 27^{-} \mathrm{T}$ cells in peripheral circulation of microfilaremics and elephan-

* Dept. of Parasitology, University of Leiden, Leiden, The Netherlands.

** Dept. of Parasitology, University of Indonesia, Jakarta, Indonesia. *** Wellcome center, Imperial College, London, U.K. 